\title{
Patient selection for cytoreductive surgery and HIPEC for the treatment of peritoneal metastases from colorectal cancer
}

This article was published in the following Dove Press journal:

Cancer Management and Research

30 June 2017

Number of times this article has been viewed

\section{Geert A Simkens \\ Koen P Rovers \\ Simon W Nienhuijs \\ Ignace $\mathrm{H}$ de Hingh}

Department of Surgical Oncology, Catharina Cancer Institute, Eindhoven, The Netherlands
Correspondence: lgnace $\mathrm{H}$ de Hingh Department of Surgery Oncology, Catharina Cancer Institute, PO Box 1350 , 5602 ZA Eindhoven, The Netherlands Tel +3I 402399111

Fax +3I 402455035 Email ignace.d.hingh@catharinaziekenhuis. $\mathrm{nl}$
Abstract: Cytoreductive surgery (CRS) and hyperthermic intraperitoneal chemotherapy (HIPEC) is a viable option for selected patients with peritoneal metastases (PM) from colorectal origin, resulting in long-term survival and even cure in some cases. However, adequate patient selection for this treatment is currently one of the major challenges. The aim of this review is to provide a comprehensive overview of clinically relevant factors associated with overall survival. This may help to guide clinicians through the complex interplay of patient, tumor, and treatment characteristics to adequately select patients who benefit the most from this extensive surgical treatment. First, basic principles of colorectal PM and the CRS and HIPEC treatment will be discussed. According to available literature, especially extent of peritoneal disease, completeness of cytoreduction, and signet ring cell histology have great influence on the outcome after CRS and HIPEC. Other factors that seem to have a negative prognostic value are the presence of liver metastases and the absence of treatment with neo-adjuvant systemic therapy. Prognostic models combining the above-mentioned factors, such as the Colorectal Peritoneal Metastases Prognostic Surgical Score nomogram, may provide clinically relevant tools to use in everyday practice.

Keywords: cytoreductive, hyperthermic intraperitoneal chemotherapy, colorectal neoplasms, peritoneal metastases, prognostic factors

\section{Introduction}

Cytoreductive surgery (CRS) and hyperthermic intraperitoneal chemotherapy (HIPEC) is an extensive surgical treatment for patients with colorectal peritoneal metastases (PM). One of the major challenges is adequate patient selection for this procedure. This review aims to give a comprehensive overview of this disease and its treatment, with special emphasis on patient selection. Therefore, the most important prognostic factors will be discussed according to available literature. This review will help to guide clinicians through a complex interplay of patient, tumor, and treatment characteristics to adequately select patients who benefit the most from this extensive surgical treatment.

\section{Colorectal cancer}

In Western countries, $\sim 15 \%$ of all cancer diagnoses have a colorectal origin. Colorectal cancer is the fourth most prevalent type of cancer and ranks second in the absolute number of estimated cancer deaths in the United States. ${ }^{1}$ Metastatic disease is the most important cause of death in colorectal cancer patients. Up to one-quarter of the patients present with synchronous stage IV disease, and another $20 \%-30 \%$ develop recurrent or systemic disease during the follow-up period after curative treatment of the primary tumor. $^{2-5}$ The liver is the most common metastatic site of colorectal cancer. ${ }^{6}$ 
The second most common manifestations are metastases to the peritoneal cavity, which have major consequences for treatment and prognosis. ${ }^{7}$

\section{PM}

PM, commonly referred to as peritoneal carcinomatosis, are metastatic deposits on the peritoneal surface throughout the abdominal cavity. These deposits may invade abdominal organs and structures, thereby frequently causing bowel obstruction, ureteral obstruction, and malignant ascites. PM may arise from virtually every primary tumor, with the most common origins being ovarian cancer in females and colorectal cancer in males. ${ }^{8}$ Other frequent origins include the stomach and pancreas, whereas the primary origin remains unknown in a substantial number of patients. ${ }^{9-11}$ Pseudomyxoma peritonei is another rare disease entity that frequently presents with peritoneal depositions. This condition is characterized by mucinous ascites and mucinous peritoneal implants, which most often originate from a ruptured low-grade mucocele of the appendix. ${ }^{12}$

In summary, PM may originate from various underlying diseases with a large variation in epidemiology, treatment strategy, and prognosis. This review will solely discuss the treatment of patients with PM from colorectal origin.

\section{Epidemiology of colorectal PM}

The incidence of synchronous PM in patients with colorectal cancer is $\sim 5 \%$, comprising $25 \%$ of all patients with synchronous stage IV disease. ${ }^{13,14}$ PM developing in the follow-up period after curative treatment of the primary colorectal tumor is called metachronous PM. Eventually, 5\% of the patients develop clinically relevant metachronous PM during the disease course. ${ }^{14-16}$ Given the PM rate of up to $40 \%$ in autopsy studies, the previously mentioned percentages are thought to be an underestimation of reality. ${ }^{17}$ This may be caused by a large group of patients without specific symptoms in whom PM were not discovered during life, probably due to the low accuracy of conventional imaging. ${ }^{18}$

\section{Risk factors for colorectal PM}

Lemmens et al have identified an advanced $\mathrm{T}$ stage, lymph node metastases, and a poor differentiation grade as independent risk factors for synchronous PM. ${ }^{13}$ Since the possibilities for preventing metachronous PM are more promising than for synchronous PM, the evidence regarding risk factors for metachronous metastases is more extensive. According to several studies, independent risk factors for metachronous $\mathrm{PM}$ are advanced $\mathrm{T}$ and $\mathrm{N}$ stage, emergency surgery, a colon versus rectal primary, and free intraperitoneal cancer cells before and/or after primary tumor resection. ${ }^{14-16,19}$ Besides these factors, mucinous adenocarcinomas and signet ring cell carcinomas tend to metastasize more frequently to the peritoneum than other histological subtypes. ${ }^{7}$

\section{Preventing metachronous colorectal PM in high-risk patients}

One of the most promising subjects of future research is the prevention of metachronous colorectal PM. Although results were biased, a recent systematic review pointed toward promising results of adjuvant intraperitoneal chemotherapy in colorectal cancer patients at high risk of PM. ${ }^{20}$ Following these results, the randomized controlled COLOPEC trial is currently including patients in the Netherlands. ${ }^{21}$ In the experimental arm of this trial, patients with pT4 or perforated colon cancer who underwent curative resection receive adjuvant treatment with HIPEC, which is thought to reduce the risk of peritoneal recurrence.

\section{Systemic treatment of colorectal PM}

For many decades, there has been little interest in investigating the treatment of colorectal PM, mainly due to the rapid progression of the disease and the lack of curative options. Curative intent surgery played a minor role in the treatment, which mainly focused on symptom relief. The efficacy of palliative systemic chemotherapy for colorectal PM remains less evident than its efficacy for other colorectal cancer metastases. ${ }^{22}$ Population-based studies reported median survival rates of up to 12 months in patients with colorectal PM who were treated with palliative systemic therapy. ${ }^{23}$ The highest currently achievable median survival in selected patients treated with systemic chemotherapy is 24 months. ${ }^{24}$ Other studies demonstrated that the addition of targeted agents to systemic chemotherapy might further improve survival. ${ }^{25,26}$ However, as the peritoneum and abdominal cavity have an impaired blood supply the efficacy of systemic therapy remains limited.

\section{CRS and HIPEC}

Based on the hypothesis that colorectal PM are a locoregional disease, a new surgical technique was first described in 1980 and further introduced by Paul Sugarbaker in the early 1990s. ${ }^{27,28}$ This multimodality treatment generally consists of two steps. First, all macroscopically visible tumor tissue is removed from the peritoneal surface by performing both peritoneal and visceral resections. This part is called CRS. 
CRS is followed by HIPEC, which is thought to eliminate the remaining microscopic tumor cells.

The effect of CRS and HIPEC in patients with colorectal PM has been investigated in two randomized controlled trials. $^{29,30}$ The first study by Verwaal et al showed a significant survival benefit in patients who underwent CRS and HIPEC followed by adjuvant systemic 5-fluorouracil with leucovorin compared to patients who received systemic 5-fluorouracil with leucovorin. However, due to this study's outdated systemic chemotherapy regimen and the lack of a CRS only control group, several questions regarding the true benefit of CRS and HIPEC remain unanswered. The randomized controlled trial by Cashin et al was terminated prematurely due to recruitment difficulties. Nevertheless, with 24 patients in each arm a significant survival benefit was found in patients treated with CRS and HIPEC compared to patients treated with oxaliplatin-based chemotherapy. Because of difficulties in conducting more randomized controlled trials, the current practice is mainly based on large retrospective cohort studies. These multicenter analyses reported median overall survival rates of up to 63 months in highly selected patients successfully treated with CRS and HIPEC. ${ }^{24,31-34}$ In a small percentage of patients, this treatment even achieved cure. ${ }^{35}$ Based on these randomized trials and cohort studies, CRS and HIPEC is currently incorporated in many guidelines worldwide. ${ }^{36}$

\section{Patient selection for CRS and HIPEC}

Although CRS and HIPEC is the standard of care in selected patients with colorectal PM in several countries, various clinical issues urgently need to be optimized to improve the outcome for these patients. While the reported median survival is $\sim 36$ months, the 1-year mortality rate and the 1-year recurrence rate after CRS and HIPEC are $13 \%$ and $35 \%$, respectively. ${ }^{37,38}$ Currently, the major challenge is to select patients for CRS and HIPEC who benefit the most from this treatment along with acceptable treatment-related morbidity and mortality. In this review, several aspects of patient selection for CRS and HIPEC as treatment for colorectal PM are discussed.

\section{Extent of peritoneal disease}

Probably, the most important and evident prognostic factor is the extent of peritoneal disease. Although several scoring systems exist, the peritoneal cancer index (PCI) score is the most commonly used and best validated. ${ }^{39}$ Numerous large cohort studies have identified the PCI score as a major prognostic factor. ${ }^{31,33,40-42}$ Goéré et al even stated that CRS and HIPEC does not seem to offer any survival benefit in patients with a PCI score of $\geq 17 .{ }^{43}$ Based on results from the aforementioned studies, surgeons should withhold performing CRS and HIPEC in patients with a PCI score $>20$. Furthermore, a closely related factor is the extent of small bowel involvement. ${ }^{44}$ CRS and HIPEC should not be performed if such a large portion of the small bowel is affected by disease that resection would result in a short bowel syndrome.

Computed tomography (CT) has a low sensitivity and specificity for detection of PM, and the radiological extent of peritoneal disease does not adequately correlate with the intraoperative PCI score. ${ }^{18}$ Improved performance may be expected from magnetic resonance imaging or from the combination of positron emission tomography and CT. ${ }^{45,46}$ Unfortunately, these imaging modalities also underestimate small peritoneal lesions, and more sensitive imaging techniques are therefore required. So far, diagnostic laparoscopy with histological confirmation remains the gold standard for diagnosing and quantifying colorectal PM, despite its more invasive character. ${ }^{47}$

\section{Completeness of cytoreduction}

The completeness of cytoreduction score measures the amount of macroscopically visible tumor that is seen after CRS. Completeness of cytoreduction is so essential that experts agree that CRS and HIPEC should only be performed if complete or nearly complete macroscopic cytoreduction is feasible. ${ }^{36}$ In a study by Verwaal et al, patients with gross residual disease of $>2.5 \mathrm{~cm}$ (R2b) had a median survival of just 5 months, compared to 17 months in patients with residual disease between $2.5 \mathrm{~mm}$ and $2.5 \mathrm{~cm}$ (R2a) and 39 months in patients with macroscopic complete cytoreduction (R1) ${ }^{48}$ Similar results were found by other large studies with a focus on prognostic factors. ${ }^{31,33,42,49,50}$ In more recent studies, the completeness of cytoreduction is less often identified as a prognostic factor, mainly because of a low number of incomplete cytoreductions. ${ }^{41}$

Since the likelihood of a complete macroscopic cytoreduction is related to a surgeon's experience, CRS and HIPEC should only be performed in specialized high-volume centers. ${ }^{51}$ When implementing this procedure in a new center, extensive training from experienced colleagues is essential.

The development of intraoperative fluorescence imaging techniques for detecting PM provides interesting possibilities for more effective cytoreduction. The general concept of these techniques is to combine a tumor-specific antibody with a fluorescence probe, thus enabling intraoperative visualization 
of tumor spots with near-infrared light. Several preclinical studies showed that these techniques have great potential for detecting PM. ${ }^{52,53}$ Clinical research is needed to confirm these promising results in the near future.

\section{Liver metastases}

For a long time, patients with combined peritoneal and liver metastases were not treated with curative intent, which at least partly attributed to the poor population-based median survival of 5 months. ${ }^{54}$ However, a curative approach may be considered in highly selected PM patients with limited and resectable hepatic disease. ${ }^{55,56}$ Consequently, over the last decade, a substantial number of studies have reported on patients who were treated with CRS and HIPEC combined with local treatment of liver metastases.

In these patients, a recently updated review of clinically heterogeneous studies revealed a median overall survival that ranged from 6 to 49 months, which was lower than in patients with isolated PM in most of the included studies. ${ }^{55}$ Additionally, a recent meta-analysis reported a significantly higher risk of death (hazard ratio 1.30) for patients with both liver metastases and PM treated with curative intent compared to patients with isolated PM. ${ }^{56}$ In addition, several retrospective cohort studies reported similar or slightly impaired survival outcomes in patients with CRS and HIPEC for PM combined with liver surgery for hepatic metastases, along with similar treatment-related morbidity. ${ }^{57-59}$

In conclusion, a patient tailored approach in patients with both peritoneal and liver metastases may result in long-term survival with acceptable morbidity. Nevertheless, survival seems to be slightly diminished compared to patients with isolated PM. Elias et al developed a tumor load-based nomogram to predict survival prior to optimal surgery in these patients, which might be of value in the complex process of decision making for this intensive treatment. ${ }^{60}$

\section{Signet ring cell carcinomas}

Generally, colorectal carcinomas are divided into three different histological subtypes; adenocarcinomas (85\%-90\%), mucinous adenocarcinomas $(10 \%-15 \%)$, and signet ring cell carcinomas (1\%). ${ }^{7}$ Regardless of treatment, the prognosis of patients with colorectal PM is strongly influenced by these histological types. ${ }^{26}$ Signet ring cell histology is particularly associated with a poor prognosis, with a median survival of $<3$ months when treated with palliative care. ${ }^{61}$

The negative prognostic impact of this histological subtype has also been described in patients who were treated with CRS and HIPEC. ${ }^{62-65}$ In several retrospective cohort series, median survival rates do not exceed 13 months after CRS and HIPEC, and 5-year survivors have not been reported. Additionally, several studies that focused on prognostic factors identified signet ring cell histology as an important negative factor with hazard ratios ranging from 2.0 to 3.7. ${ }^{41,48,56,66}$

Nevertheless, with respect to palliative care, a similar relative survival gain can be achieved by CRS and HIPEC in patients with signet ring cell histology compared with patients with adenocarcinomas and mucinous adenocarcinomas. ${ }^{61}$ The authors from this population-based study conclude that patients with signet ring cell carcinomas benefit from CRS and HIPEC. Since patients with these carcinomas are often young, an aggressive surgical approach may be a realistic option in a highly selected subgroup.

\section{Rectal origin of PM}

In non-metastasized patients, colon and rectal cancer are considered as separate entities with a different treatment and prognosis. ${ }^{67,68}$ In peritoneally metastasized patients who are treated with CRS and HIPEC, these differences are less evident. Colorectal PM are often considered as one disease, regardless of their colon or rectal origin. As a result, the large group of colon cancer patients often camouflages the results of the small portion of rectal cancer patients.

The available evidence that reported on survival rates of patients with rectal PM treated with CRS and HIPEC is contradictory and consists of retrospective cohort studies. The most recent and largest study included 29 rectal cancer patients and reported similar recurrence and survival rates compared with colon cancer patients, with 5-year survival rates of $\sim 30 \%$ in both groups. ${ }^{69}$ Similar results were found in two smaller retrospective studies. ${ }^{70,71}$ These results are in contrast with two studies with rectal cancer patients who were treated with CRS and HIPEC ( $\mathrm{n}=5$ and 11), in which survival was diminished compared to colon PM patients. ${ }^{48,72}$ In large studies that investigated prognostic factors for survival after CRS and HIPEC, a rectal origin did not seem to influence survival. ${ }^{33,40,56,73}$ Even in selected cases of locally advanced rectal cancer with synchronous $\mathrm{PM}$, long-term survival and acceptable morbidity were achieved with a combination of CRS and HIPEC and intraoperative radiotherapy. ${ }^{74}$ In conclusion, CRS and HIPEC is a feasible option in selected patients with PM from rectal cancer, with similar outcomes as patients with PM of colon cancer.

\section{Neo-adjuvant systemic treatment}

The value of neo-adjuvant systemic therapy remains controversial due to an absence of randomized studies. Several 
retrospective observational studies analyzed survival outcomes of patients who were stratified for neo-adjuvant versus no neo-adjuvant systemic therapy. ${ }^{31,33,35,75-78}$ However, results of studies on this topic are hardly interpretable due to clinical heterogeneity, selected populations, and the absence of details on systemic regimens. ${ }^{86}$ Therefore, available evidence does not allow for definitive conclusions and recommendations.

Hypothetically, neo-adjuvant systemic therapy may increase the chance of achieving a complete cytoreduction by preoperative tumor downsizing. However, a pooled subgroup analysis of randomized studies in advanced colorectal cancer endorses the dogma that colorectal PM are relatively resistant to systemic therapy compared to other isolated sites of metastases. ${ }^{22}$ By using Blazer's classification, Passot et al reported a complete and major pathological response rate of $10 \%$ and $20 \%$, which was lower than the reported pathological response rates of colorectal liver metastases. ${ }^{79-81}$ To date, no studies have prospectively investigated the pathological tumor response of colorectal PM to neo-adjuvant systemic therapy. Three single arm Phase II studies are currently investigating the safety, feasibility, and efficacy of neo-adjuvant FOLFOX with bevacizumab (BEV-IP trial, NCT02399410), FOLFOXIRI with bevacizumab (CARCINOSIS trial, NCT02591667), and FOLFOX/FOLFIRI with cetuximab (COMBATAC trial, NCT01540344) prior to CRS and HIPEC for potentially resectable colorectal PM. Results of these studies will provide more insight in the sensitivity of colorectal PM to modern neo-adjuvant chemotherapy with targeted agents.

Besides preoperative tumor downsizing, improved patient selection is another potential and more commonly accepted advantage of neo-adjuvant systemic therapy. It may be postulated that patients with disease progression upon neo-adjuvant systemic therapy do not benefit from CRS and HIPEC due to aggressive tumor biology. A French study reported an impressive median overall survival of 63 months in selected patients who received CRS and HIPEC after they revealed a favorable tumor response upon neoadjuvant systemic therapy. ${ }^{24}$ To compare, median survival was 39 months in another cohort that received upfront CRS and HIPEC intentionally followed by adjuvant systemic therapy. ${ }^{77}$ However, nothing is known about the number of patients that do not qualify for CRS and HIPEC due to disease progression or severe toxicity upon neo-adjuvant systemic therapy, thereby impeding an intention-to-treat comparison between these treatment strategies. For example, an intention-to-treat analysis in resectable colorectal liver metastases revealed no overall survival difference of perioperative systemic therapy and surgery compared to upfront surgery. ${ }^{82}$ Given the absence of such studies in colorectal PM, the CAIRO6 trial (NCT02758951) will soon start to randomize patients with potentially resectable colorectal PM between upfront CRS and HIPEC and CRS and HIPEC with perioperative systemic therapy consisting of neo-adjuvant FOLFOX with bevacizumab.

Taken together, neo-adjuvant systemic therapy may improve survival after CRS and HIPEC by improving patient selection, but its benefit on an intention-to-treat basis needs to be confirmed by results of ongoing and future studies.

\section{Prognostic models to predict survival}

This review discussed several important factors for overall survival after CRS and HIPEC. Ideally, these factors are combined in a prognostic model to predict survival of colorectal PM patients treated with curative intent. Indeed, several prognostic scores have been published. Verwaal et al were the first to combine location of the primary tumor, histological subtype, and extent of peritoneal disease into a prognostic model. ${ }^{48}$ However, to the knowledge of the authors, this statistically sound model has never been externally validated or extensively used in clinical practice.

To date, the Peritoneal Surface Disease Severity Score (PSDSS) is the most frequently evaluated model for colorectal PM patients. ${ }^{6}{ }^{6}$ It was developed by Pelz et al and includes the preoperative CT scan-based PCI score, histological subtype, lymph node status, and clinical symptoms. The development study only included 40 patients and did not seem to use regression coefficients to determine the weighed scores. Several multi-institutional studies evaluated the prognostic value of PSDSS and agreed that it has some predictive value. ${ }^{32,83,84}$ Nevertheless, none of these studies assessed the predictive value of PSDSS according to validated model performance measures such as the Harrel's C index or Nagelkerke $R^{2}$ statistic. Additionally, PCI appeared to be superior to PSDSS in predicting overall and disease-free survival in colorectal PM patients treated with CRS and HIPEC. ${ }^{85}$

A recent study externally validated the PSDSS, and its performance was suboptimal with a Harrel's C index of 0.62 and a Nagelkerke $R^{2}$ statistic of $0.08 .{ }^{41}$ Subsequently, the authors developed a new prognostic score named Colorectal Peritoneal Metastases Prognostic Surgical Score (COMPASS). This Cox regression-based nomogram included four factors: age, PCI score, lymph node status, and signet ring cell histology. With a Harrel's $C$ index of 0.72 and a Nagelkerke $R^{2}$ statistic of 0.19 , it performed considerably better than the PSDSS. Future research focusing on external validation of 
the new COMPASS model is warranted and will give more insight into the possibilities of this score for colorectal PM patients treated with CRS and HIPEC.

\section{Conclusion}

CRS and HIPEC is a viable option for selected patients with PM from colorectal origin, resulting in long-term survival and even cure in some patients. However, adequate patient selection for this treatment is currently one of the major challenges. This review focused on several important issues in this complex interplay of patient, tumor, and treatment characteristics. According to available literature, especially extent of peritoneal disease, completeness of cytoreduction, and signet ring cell histology have great influence on the outcome after CRS and HIPEC. The presence of liver metastases seems to have a negative prognostic impact. In contrast, treatment with neo-adjuvant systemic therapy seems to prolong survival after CRS and HIPEC. Additionally, rectal cancer should not be regarded as a strong negative prognostic factor. In general, only patients with limited peritoneal disease, eligible for complete macroscopic cytoreduction and without signet ring cell histology, are able to achieve long-term survival after CRS and HIPEC. Prognostic models combining the above-mentioned factors, such as the COMPASS nomogram, may provide clinically relevant tools to use in everyday practice.

\section{Disclosure}

The authors report no conflicts of interest in this work.

\section{References}

1. American Cancer Society. Cancer Statistics Center. Available at: https:// cancerstatisticscenter.cancer.org/. Accessed August 1, 2016.

2. Lemmens V, van Steenbergen L, Janssen-Heijnen M, Martijn H, Rutten H, Coebergh JW. Trends in colorectal cancer in the south of the Netherlands 1975-2007: rectal cancer survival levels with colon cancer survival. Acta Oncol. 2010;49(6):784-796.

3. van der Geest LG, Lam-Boer J, Koopman M, Verhoef C, Elferink MA, de Wilt JH. Nationwide trends in incidence, treatment and survival of colorectal cancer patients with synchronous metastases. Clin Exp Metastasis. 2015;32(5):457-465.

4. Manfredi S, Bouvier AM, Lepage C, Hatem C, Dancourt V, Faivre J. Incidence and patterns of recurrence after resection for cure of colonic cancer in a well defined population. Br J Surg. 2006;93(9):1115-1122.

5. van Gestel YR, de Hingh IH, van Herk-Sukel MP, et al. Patterns of metachronous metastases after curative treatment of colorectal cancer. Cancer Epidemiol. 2014;38(4):448-454.

6. Hess KR, Varadhachary GR, Taylor SH, et al. Metastatic patterns in adenocarcinoma. Cancer. 2006;106(7):1624-1633.

7. Hugen N, van de Velde CJ, de Wilt JH, Nagtegaal ID. Metastatic pattern in colorectal cancer is strongly influenced by histological subtype. Ann Oncol. 2014;25(3):651-657.

8. Hennessy BT, Coleman RL, Markman M. Ovarian cancer. Lancet. 2009;374(9698):1371-1382.
9. Thomassen I, Lemmens VE, Nienhuijs SW, Luyer MD, Klaver YL, de Hingh $\mathrm{IH}$. Incidence, prognosis, and possible treatment strategies of peritoneal carcinomatosis of pancreatic origin: a population-based study. Pancreas. 2013;42(1):72-75.

10. Thomassen I, van Gestel YR, van Ramshorst B, et al. Peritoneal carcinomatosis of gastric origin: a population-based study on incidence, survival and risk factors. Int J Cancer. 2014;134(3):622-628.

11. Thomassen I, Verhoeven RH, van Gestel YR, van de Wouw AJ, Lemmens VE, de Hingh IH. Population-based incidence, treatment and survival of patients with peritoneal metastases of unknown origin. Eur J Cancer. 2014;50(1):50-56.

12. Moran B, Baratti D, Yan TD, Kusamura S, Deraco M. Consensus statement on the loco-regional treatment of appendiceal mucinous neoplasms with peritoneal dissemination (pseudomyxoma peritonei). J Surg Oncol. 2008;98(4):277-282.

13. Lemmens VE, Klaver YL, Verwaal VJ, Rutten HJ, Coebergh JW, de Hingh IH. Predictors and survival of synchronous peritoneal carcinomatosis of colorectal origin: a population-based study. Int J Cancer. 2011;128(11):2717-2725.

14. Segelman J, Granath F, Holm T, Machado M, Mahteme H, Martling A. Incidence, prevalence and risk factors for peritoneal carcinomatosis from colorectal cancer. Br J Surg. 2012;99(5):699-705.

15. Kerscher AG, Chua TC, Gasser M, et al. Impact of peritoneal carcinomatosis in the disease history of colorectal cancer management: a longitudinal experience of 2406 patients over two decades. Br J Cancer. 2013;108(7):1432-1439.

16. Segelman J, Akre O, Gustafsson UO, Bottai M, Martling A. Individualized prediction of risk of metachronous peritoneal carcinomatosis from colorectal cancer. Colorectal Dis. 2014;16(5):359-367.

17. Koppe MJ, Boerman OC, Oyen WJ, Bleichrodt RP. Peritoneal carcinomatosis of colorectal origin: incidence and current treatment strategies. Ann Surg. 2006;243(2):212-222.

18. Koh JL, Yan TD, Glenn D, Morris DL. Evaluation of preoperative computed tomography in estimating peritoneal cancer index in colorectal peritoneal carcinomatosis. Ann Surg Oncol. 2009;16(2):327-333.

19. Rekhraj S, Aziz O, Prabhudesai S, et al. Can intra-operative intraperitoneal free cancer cell detection techniques identify patients at higher recurrence risk following curative colorectal cancer resection: a metaanalysis. Ann Surg Oncol. 2008;15(1):60-68.

20. Sloothaak DA, Mirck B, Punt CJ, et al. Intraperitoneal chemotherapy as adjuvant treatment to prevent peritoneal carcinomatosis of colorectal cancer origin: a systematic review. Br JCancer: 2014;111(6):1112-1121.

21. Klaver CE, Musters GD, Bemelman WA, et al. Adjuvant hyperthermic intraperitoneal chemotherapy (HIPEC) in patients with colon cancer at high risk of peritoneal carcinomatosis; the COLOPEC randomized multicentre trial. BMC Cancer. 2015;15:428.

22. Franko J, Shi Q, Meyers JP, et al. Prognosis of patients with peritoneal metastatic colorectal cancer given systemic therapy: an analysis of individual patient data from prospective randomised trials from the Analysis and Research in Cancers of the Digestive System (ARCAD) database. Lancet Oncol. 2016;17(12):1709-1719.

23. Razenberg LG, van Gestel YR, Creemers GJ, Verwaal VJ, Lemmens VE, de Hingh IH. Trends in cytoreductive surgery and hyperthermic intraperitoneal chemotherapy for the treatment of synchronous peritoneal carcinomatosis of colorectal origin in the Netherlands. Eur J Surg Oncol. 2015;41(4):466-471.

24. Elias D, Lefevre JH, Chevalier J, et al. Complete cytoreductive surgery plus intraperitoneal chemohyperthermia with oxaliplatin for peritoneal carcinomatosis of colorectal origin. J Clin Oncol. 2009;27(5):681-685.

25. Klaver YL, Simkens LH, Lemmens VE, et al. Outcomes of colorectal cancer patients with peritoneal carcinomatosis treated with chemotherapy with and without targeted therapy. Eur J Surg Oncol. 2012;38(7):617-623.

26. Razenberg LG, van Gestel YR, Lemmens VE, de Wilt JH, Creemers GJ, de Hingh IH. The prognostic relevance of histological subtype in patients with peritoneal metastases from colorectal cancer: a nationwide population-based study. Clin Colorectal Cancer. 2015;14(4):e13-e19. 
27. Spratt JS, Adcock RA, Muskovin M, Sherrill W, McKeown J. Clinical delivery system for intraperitoneal hyperthermic chemotherapy. Cancer Res. 1980;40(2):256-260.

28. Sugarbaker PH. Peritonectomy procedures. Ann Surg. 1995;221(1):29-42.

29. Cashin PH, Mahteme H, Spang N, et al. Cytoreductive surgery and intraperitoneal chemotherapy versus systemic chemotherapy for colorectal peritoneal metastases: a randomised trial. Eur J Cancer. 2016;53: $155-162$.

30. Verwaal VJ, van Ruth S, de Bree E, et al. Randomized trial of cytoreduction and hyperthermic intraperitoneal chemotherapy versus systemic chemotherapy and palliative surgery in patients with peritoneal carcinomatosis of colorectal cancer. J Clin Oncol. 2003;21(20): 3737-3743.

31. Elias D, Gilly F, Boutitie F, et al. Peritoneal colorectal carcinomatosis treated with surgery and perioperative intraperitoneal chemotherapy: retrospective analysis of 523 patients from a multicentric French study. J Clin Oncol. 2010;28(1):63-68.

32. Esquivel J, Lowy AM, Markman M, et al. The American Society of Peritoneal Surface Malignancies (ASPSM) multi-institution evaluation of the peritoneal surface disease severity score (PSDSS) in 1,013 patients with colorectal cancer with peritoneal carcinomatosis. Ann Surg Oncol. 2014;21(13):4195-4201.

33. Glehen O, Kwiatkowski F, Sugarbaker PH, et al. Cytoreductive surgery combined with perioperative intraperitoneal chemotherapy for the management of peritoneal carcinomatosis from colorectal cancer: a multi-institutional study. J Clin Oncol. 2004;22(16):3284-3292.

34. Kuijpers AM, Mirck B, Aalbers AG, et al. Cytoreduction and HIPEC in the Netherlands: nationwide long-term outcome following the Dutch protocol. Ann Surg Oncol. 2013;20(13):4224-4230.

35. Goere D, Malka D, Tzanis D, et al. Is there a possibility of a cure in patients with colorectal peritoneal carcinomatosis amenable to complete cytoreductive surgery and intraperitoneal chemotherapy? Ann Surg. 2013;257(6):1065-1071.

36. Esquivel J, Piso P, Verwaal V, et al. American Society of Peritoneal Surface Malignancies opinion statement on defining expectations from cytoreductive surgery and hyperthermic intraperitoneal chemotherapy in patients with colorectal cancer. J Surg Oncol. 2014;110(7): 777-778.

37. Simkens GA, van Oudheusden TR, Braam HJ, et al. Treatment-related mortality after cytoreductive surgery and HIPEC in patients with colorectal peritoneal carcinomatosis is underestimated by conventional parameters. Ann Surg Oncol. 2016;23(1):99-105.

38. Simkens GA, van Oudheusden TR, Luyer MD, et al. Serious postoperative complications affect early recurrence after cytoreductive surgery and HIPEC for colorectal peritoneal carcinomatosis. Ann Surg Oncol. 2015;22(8):2656-2662.

39. Jacquet P, Sugarbaker PH. Clinical research methodologies in diagnosis and staging of patients with peritoneal carcinomatosis. Cancer Treat Res. 1996;82:359-374

40. da Silva RG, Sugarbaker PH. Analysis of prognostic factors in seventy patients having a complete cytoreduction plus perioperative intraperitoneal chemotherapy for carcinomatosis from colorectal cancer. $\mathrm{J} \mathrm{Am}$ Coll Surg. 2006;203(6):878-886.

41. Simkens GA, van Oudheusden TR, Nieboer D, et al. Development of a prognostic nomogram for patients with peritoneally metastasized colorectal cancer treated with cytoreductive surgery and HIPEC. Ann Surg Oncol. 2016;23(13):4214-4221.

42. Cavaliere F, De Simone M, Virzi S, et al. Prognostic factors and oncologic outcome in 146 patients with colorectal peritoneal carcinomatosis treated with cytoreductive surgery combined with hyperthermic intraperitoneal chemotherapy: Italian multicenter study S.I.T.I.L.O. Eur $J$ Surg Oncol. 2011;37(2):148-154.

43. Goere D, Souadka A, Faron M, et al. Extent of colorectal peritoneal carcinomatosis: attempt to define a threshold above which HIPEC does not offer survival benefit: a comparative study. Ann Surg Oncol. 2015;22(9):2958-2964.
44. Elias D, Mariani A, Cloutier AS, et al. Modified selection criteria for complete cytoreductive surgery plus HIPEC based on peritoneal cancer index and small bowel involvement for peritoneal carcinomatosis of colorectal origin. Eur J Surg Oncol. 2014;40(11):1467-1473.

45. Soussan M, Des Guetz G, Barrau V, et al. Comparison of FDG-PET/ $\mathrm{CT}$ and MR with diffusion-weighted imaging for assessing peritoneal carcinomatosis from gastrointestinal malignancy. Eur Radiol. 2012;22(7):1479-1487.

46. Li J, Yan R, Lei J, Jiang C. Comparison of PET with PET/CT in detecting peritoneal carcinomatosis: a meta-analysis. Abdom Imaging. 2015;40(7):2660-2666.

47. Valle M, Garofalo A. Laparoscopic staging of peritoneal surface malignancies. Eur J Surg Oncol. 2006;32(6):625-627.

48. Verwaal VJ, van Tinteren H, van Ruth S, Zoetmulder FA. Predicting the survival of patients with peritoneal carcinomatosis of colorectal origin treated by aggressive cytoreduction and hyperthermic intraperitoneal chemotherapy. Br J Surg. 2004;91(6):739-746.

49. Glehen O, Gilly FN, Boutitie F, et al. Toward curative treatment of peritoneal carcinomatosis from nonovarian origin by cytoreductive surgery combined with perioperative intraperitoneal chemotherapy: a multiinstitutional study of 1,290 patients. Cancer. 2010;116(24):5608-5618.

50. Shen P, Hawksworth J, Lovato J, et al. Cytoreductive surgery and intraperitoneal hyperthermic chemotherapy with mitomycin $\mathrm{C}$ for peritoneal carcinomatosis from nonappendiceal colorectal carcinoma. Ann Surg Oncol. 2004;11(2):178-186.

51. Kuijpers AM, Aalbers AG, Nienhuijs SW, et al. Implementation of a standardized HIPEC protocol improves outcome for peritoneal malignancy. World J Surg. 2015;39(2):453-460.

52. Asanuma D, Sakabe M, Kamiya M, et al. Sensitive beta-galactosidasetargeting fluorescence probe for visualizing small peritoneal metastatic tumours in vivo. Nat Commun. 2015;6:6463.

53. Boonstra MC, van Driel PB, van Willigen DM, et al. uPAR-targeted multimodal tracer for pre- and intraoperative imaging in cancer surgery. Oncotarget. 2015;6(16):14260-14273.

54. Thomassen I, van Gestel YR, Lemmens VE, de Hingh IH. Incidence, prognosis, and treatment options for patients with synchronous peritoneal carcinomatosis and liver metastases from colorectal origin. Dis Colon Rectum. 2013;56(12):1373-1380.

55. El-Nakeep S, Rashad N, Oweira H, et al. Intraperitoneal chemotherapy and cytoreductive surgery for peritoneal metastases coupled with curative treatment of colorectal liver metastases: an updated systematic review. Expert Rev Gastroenterol Hepatol. 2017;11(3):249-258.

56. Kwakman R, Schrama AM, van Olmen JP, et al. Clinicopathological parameters in patient selection for cytoreductive surgery and hyperthermic intraperitoneal chemotherapy for colorectal cancer metastases: a meta-analysis. Ann Surg. 2016;263(6):1102-1111.

57. Lorimier G, Linot B, Paillocher N, et al. Curative cytoreductive surgery followed by hyperthermic intraperitoneal chemotherapy in patients with peritoneal carcinomatosis and synchronous resectable liver metastases arising from colorectal cancer. Eur J Surg Oncol. 2017;43(1):150-158.

58. Navez J, Remue C, Leonard D, et al. Surgical treatment of colorectal cancer with peritoneal and liver metastases using combined liver and cytoreductive surgery and hyperthermic intraperitoneal chemotherapy: report from a single-centre experience. Ann Surg Oncol. 2016;23(Suppl 5): 666-673.

59. Randle RW, Doud AN, Levine EA, et al. Peritoneal surface disease with synchronous hepatic involvement treated with cytoreductive surgery (CRS) and hyperthermic intraperitoneal chemotherapy (HIPEC). Ann Surg Oncol. 2015;22(5):1634-1638.

60. Elias D, Faron M, Goere D, et al. A simple tumor load-based nomogram for surgery in patients with colorectal liver and peritoneal metastases. Ann Surg Oncol. 2014;21(6):2052-2058.

61. Simkens GA, Razenberg LG, Lemmens VE, Rutten HJ, Creemers GJ, de Hingh IH. Histological subtype and systemic metastases strongly influence treatment and survival in patients with synchronous colorectal peritoneal metastases. Eur J Surg Oncol. 2016;42(6):794-800. 
62. van Oudheusden TR, Braam HJ, Nienhuijs SW, et al. Poor outcome after cytoreductive surgery and HIPEC for colorectal peritoneal carcinomatosis with signet ring cell histology. J Surg Oncol. 2015;111(2):237-242.

63. Winer J, Zenati M, Ramalingam L, et al. Impact of aggressive histology and location of primary tumor on the efficacy of surgical therapy for peritoneal carcinomatosis of colorectal origin. Ann Surg Oncol. 2014;21(5): 1456-1462.

64. Chua TC, Pelz JO, Kerscher A, Morris DL, Esquivel J. Critical analysis of 33 patients with peritoneal carcinomatosis secondary to colorectal and appendiceal signet ring cell carcinoma. Ann Surg Oncol. 2009;16(10):2765-2770.

65. Van Sweringen HL, Hanseman DJ, Ahmad SA, Edwards MJ, Sussman JJ. Predictors of survival in patients with high-grade peritoneal metastases undergoing cytoreductive surgery and hyperthermic intraperitoneal chemotherapy. Surgery. 2012;152(4):617-624.

66. Pelz JO, Stojadinovic A, Nissan A, Hohenberger W, Esquivel J. Evaluation of a peritoneal surface disease severity score in patients with colon cancer with peritoneal carcinomatosis. J Surg Oncol. 2009;99(1):9-15.

67. van Erning FN, van Steenbergen LN, Lemmens VE, et al. Conditional survival for long-term colorectal cancer survivors in the Netherlands: who do best? Eur J Cancer. 2014;50(10):1731-1739.

68. Van Leersum NJ, Snijders HS, Henneman D, et al. The Dutch surgical colorectal audit. Eur J Surg Oncol. 2013;39(10):1063-1070.

69. Simkens GA, van Oudheusden TR, Braam HJ, et al. Cytoreductive surgery and HIPEC offers similar outcomes in patients with rectal peritoneal metastases compared to colon cancer patients: a matched case control study. J Surg Oncol. 2016;113(5):548-553.

70. Ung L, Chua TC, Morris DL. Peritoneal metastases of lower gastrointestinal tract origin: a comparative study of patient outcomes following cytoreduction and intraperitoneal chemotherapy. J Cancer Res Clin Oncol. 2013;139(11):1899-1908.

71. Votanopoulos KI, Swett K, Blackham AU, et al. Cytoreductive surgery with hyperthermic intraperitoneal chemotherapy in peritoneal carcinomatosis from rectal cancer. Ann Surg Oncol. 2013;20(4):1088-1092.

72. Gomes da Silva R, Cabanas J, Sugarbaker PH. Limited survival in the treatment of carcinomatosis from rectal cancer. Dis Colon Rectum. 2005;48(12):2258-2263.

73. Rivard JD, McConnell YJ, Temple WJ, Mack LA. Cytoreduction and heated intraperitoneal chemotherapy for colorectal cancer: are we excluding patients who may benefit? J Surg Oncol. 2014;109(2):104-109.

74. Klaver YL, Lemmens VE, Nienhuijs SW, Nieuwenhuijzen GA, Rutten $\mathrm{HJ}$, de Hingh IH. Intraoperative radiotherapy and cytoreductive surgery with hyperthermic intraperitoneal chemotherapy. Five consecutive case reports of locally advanced rectal cancer with synchronous peritoneal carcinomatosis. Strahlenther Onkol. 2013;189(3):256-260.
75.Passot G, Vaudoyer D, Cotte E, et al. Progression following neoadjuvant systemic chemotherapy may not be a contraindication to a curative approach for colorectal carcinomatosis. Ann Surg. 2012;256(1):125-129.

76. Baratti D, Kusamura S, Iusco D, et al. Postoperative complications after cytoreductive surgery and hyperthermic intraperitoneal chemotherapy affect long-term outcome of patients with peritoneal metastases from colorectal cancer: a two-center study of 101 patients. Dis Colon Rectum. 2014;57(7):858-868

77. Devilee RA, Simkens GA, van Oudheusden TR, et al. Increased survival of patients with synchronous colorectal peritoneal metastases receiving preoperative chemotherapy before cytoreductive surgery and hyperthermic intraperitoneal chemotherapy. Ann Surg Oncol. 2016;23(9):2841-2848.

78. Ceelen W, Van Nieuwenhove Y, Putte DV, Pattyn P. Neoadjuvant chemotherapy with bevacizumab may improve outcome after cytoreduction and hyperthermic intraperitoneal chemoperfusion (HIPEC) for colorectal carcinomatosis. Ann Surg Oncol. 2014;21(9):3023-3028.

79. Passot G, You B, Boschetti G, et al. Pathological response to neoadjuvant chemotherapy: a new prognosis tool for the curative management of peritoneal colorectal carcinomatosis. Ann Surg Oncol. 2014;21(8):2608-2614.

80. Blazer DG 3rd, Kishi Y, Maru DM, et al. Pathologic response to preoperative chemotherapy: a new outcome end point after resection of hepatic colorectal metastases. J Clin Oncol. 2008;26(33):5344-5351.

81. Chan G, Hassanain M, Chaudhury P, et al. Pathological response grade of colorectal liver metastases treated with neoadjuvant chemotherapy. HPB (Oxford). 2010;12(4):277-284.

82. Nordlinger B, Sorbye H, Glimelius B, et al. Perioperative chemotherapy with FOLFOX4 and surgery versus surgery alone for resectable liver metastases from colorectal cancer (EORTC intergroup trial 40983): a randomised controlled trial. Lancet. 2008;371(9617):1007-1016.

83. Chua TC, Morris DL, Saxena A, et al. Influence of modern systemic therapies as adjunct to cytoreduction and perioperative intraperitoneal chemotherapy for patients with colorectal peritoneal carcinomatosis: a multicenter study. Ann Surg Oncol. 2011;18(6):1560-1567.

84. Prada-Villaverde A, Esquivel J, Lowy AM, et al. The American Society of Peritoneal Surface Malignancies evaluation of HIPEC with mitomycin $\mathrm{C}$ versus oxaliplatin in 539 patients with colon cancer undergoing a complete cytoreductive surgery. J Surg Oncol. 2014;110(7):779-785.

85. Ng JL, Ong WS, Chia CS, Tan GH, Soo KC, Teo MC. Prognostic relevance of the peritoneal surface disease severity score compared to the peritoneal cancer index for colorectal peritoneal carcinomatosis. Int J Surg Oncol. 2016;2016:2495131.

86. Rovers KP, Simkens GA, Punt CJ, van Dieren S, Tanis PJ, de Hingh IH. Perioperative systemic therapy for resectable colorectal peritoneal metastases: Sufficient evidence for its widespread use? A critical systematic review. Crit Rev Oncol Hematol. 2017;114:53-62.
Cancer Management and Research

\section{Publish your work in this journal}

Cancer Management and Research is an international, peer-reviewed open access journal focusing on cancer research and the optimal use of preventative and integrated treatment interventions to achieve improved outcomes, enhanced survival and quality of life for the cancer patient. The manuscript management system is completely online and includes

\section{Dovepress}

a very quick and fair peer-review system, which is all easy to use. Visit $\mathrm{http}: / / \mathrm{www}$.dovepress.com/testimonials.php to read real quotes from published authors. 